\title{
Internalization and effects on cellular ultrastructure of nickel nanoparticles in rat kidneys
}

This article was published in the following Dove Press journal:

International Journal of Nanomedicine

\section{Shang Ziyad Abdulqadir \\ Falah Mohammad Aziz}

Biology Department, Salahaddin UniversityErbil, Erbil, Kurdistan Region, Iraq
Correspondence: Falah Mohammad Aziz Department of Biology, College of Science, Salahaddin University-Erbil, Erbil, Kurdistan Region 44002, Iraq

Tel +964750466 5531

Email falah.aziz@su.edu.krd

\begin{abstract}
Purpose: Since nanoparticles (NPs) are beginning to be introduced in medicine and industry, it is mendatory to evaluate their biological side-effects, among other things. The present study aimed to investigate the pathways by which nickel nanoparticles (NiNPs) enter nephrons and to evaluate their localization and effects on cellular ultrastructure.

Methods: Rats were injected intraperitoneally with $20 \mathrm{~nm} \mathrm{NiNPs}(20 \mathrm{mg} / \mathrm{Kg} / \mathrm{b} . w . /$ day $)$ for 28 consecutive days. Transmission electron microscope technique was used to detect localization of NiNPs and their effects on cellular ultrastructure in rat kidneys. Additionally, measurements of certain biochemical parameters such as creatinine, urea, uric acid and phosphorus for investigating renal function following NiNPs treatment were taken.
\end{abstract}

Results: The presence of NiNPs in the nephrons in treated rats was confirmed by transmission electron microscopy. NiNPs entered the renal tubules cells via various pathways. The results indicated that NiNPs administration induced ultrastructural changes in the proximal cells of renal tubules and certain glomerular cells (podocytes and mesangial cells). Additionally, NiNPs were found to be localized in the mitochondria, which led to a significant decrease in their density and morphology. Furthermore, cell death was induced in the glomerular cells as found with a Terminal deoxynucleotidyl transferase dUTP Nick End Labeling (TUNEL) assay and through detection of p35 using immunohistochemical staining.

Conclusion: Herein, NiNPs were found to induce various cellular ultrastructural changes in the kidneys of rats. NiNPs used diverse pathways to internalize into the cytoplasm of the proximal convoluted tubules (PT) cells across the basement membrane, and also through the plasma membrane of two adjacent PT cells. NiNPs internalization, accumulation and their alterations of the cellular ultrastructure affected rat renal function.

Keywords: Nickel nanoparticles, internalization, ultrastructure, proximal convoluted tubules, podocytes, mesangial cells

\section{Introduction}

Nanoparticles (NPs) are particles that have dimensions equivalent to $100 \mathrm{~nm}$ or less. ${ }^{1}$ The increased use of NPs in the industry and in biomedical applications has led to increased public concern regarding the biological and medical effects of NPs. ${ }^{2}$ Over the past several decades, nickel nanoparticles (NiNPs) have been widely used in hydrogen storages, as chemical catalysts, in ceramic capacitors, in sensors and conductive paint, and in nanomedicine. ${ }^{3}$ Nanoparticles translocate across the plasma membrane via endocytosis using different direct permeation pathways ${ }^{1}$ that depend on their physicochemical properties. ${ }^{4}$ Translocation of NPs across the cell membrane is a requirement during drug delivery and nanotoxicological research, however, the 
fate of these particles following their internalization and the mechanisms of their eventual elimination require further investigation. ${ }^{5}$

The main mechanism suggested for NiNPs-induced cell damage is via induction of increased levels of reactive oxygen species (ROS), which are markers of oxidative stress, ${ }^{6-10}$ this has also been found to be a key mechanism for the cytotoxicity of other nanomaterials. ${ }^{11-13}$ NiNPs were found to induce oxidative DNA damage, ${ }^{14}$ inflammation, cell degeneration, ${ }^{9}$ cell cycle arrest, ${ }^{10}$ cytogenetic alterations and apoptosis. $^{15}$

Kidneys are one of the main organs where there is accumulation of nanoparticles, particularly NiNPs. ${ }^{16}$ The kidneys of rats injected with nanoparticles were previously shown to display various histopathological changes such as vacuolation and pyknosis in renal tubular epithelial cells and glomerular damage. ${ }^{17-19}$ Nickel ions have also found to induce significant kidney damage, as well as lead to reduced activities of enzymatic and non-enzymatic antioxidants. ${ }^{20}$

In the present study, we investigated the internalization and localization of NiNPs and their effects on the cellular ultrastructure in rat kidneys. NiNPs induced cellular ultrastructural alterations in the proximal cells of the renal tubules and certain glomerular cells. NiNPs led to a remarkable reduction in the density and morphology of mitochondria. Interestingly, NiNPs significantly enhanced apoptosis/cell death in the cells of the rat kidneys.

\section{Material and methods}

\section{Material}

Powdered $20 \mathrm{~nm}$ nickel nanoparticles (NiNPs) were purchased from Sigma-Aldrich Co. (St Louis, MO, USA). All other chemicals were of laboratory grade and were used as received.

\section{Preparation of NINPs suspension}

A stock suspension of $20 \mathrm{~nm}$ NiNPs was prepared in normal saline $(10 \mathrm{mg} \backslash \mathrm{mL})$ by sonication for 30 seconds in an ultrasonic homogenizer (model $150 \mathrm{VT}$, manufactured by biologica, Inc., Manassas, VA, USA); the particle suspensions were kept on ice for 15 seconds and sonicated again on ice for a total of 3 minutes at a power of $400 \mathrm{~W}$. The NiNPs were vibrated for 2 minutes, immediately prior to the eventual injection. A concentration of $20 \mathrm{mg} / \mathrm{kg}$ body weight was prepared from this stock suspension. The morphology of the NiNPs was examined in images taken by FEI Tecnai Spirit 10 Transmission Electron Microscope (TEM).

\section{Animals}

Twelve 12-week-old male Wistar rats weighing 200-220 $\mathrm{g}$ were obtained from the animal house of Salahaddin University-Erbil. Animals were maintained in stainless steel mesh cages with free access to tap water and pellets. Moreover, they were maintained at a mean temperature of $21 \pm 2{ }^{\circ} \mathrm{C}$ and a mean relative humidity of $35 \%$. The experiment was conducted following the protocols approved by the Animal Care Ethical Committee of College of Science, Salahaddin University-Erbil, under the number 4N/115 on April 5, 2017 according to the Institutional Animal Care and Use Committee (IACUC) guidelines for animal care $^{21}$ and UBC Animal Care Guidelines for intraperitoneal injections. ${ }^{22}$

\section{Experimental design}

The 12 rats were equally and randomly divided into two groups (kept in two separate cages); each group contained six animals. In group 1 (control group); the rats received the vehicle, while in group 2 the rats received $20 \mathrm{~nm}$ NiNPs $(20 \mathrm{mg} / \mathrm{kg}$ ) for 28 consecutive days. A needle gauge that was $22 \mathrm{~g}$ was used for the intraperitoneal injection. The selected NiNPs dose was based on previous studies. ${ }^{23,24}$ Then, 24 hours after the last treatment, all rats were sacrificed and kidney samples were collected for the cellular ultrastructure analysis.

\section{Electron microscopy}

Kidney tissues $\left(\leq 1 \mathrm{~mm}^{3}\right)$ were fixed in $2.5 \%$ glutaraldehyde in $0.1 \mathrm{M}$ cacodylate buffer $\mathrm{pH} 7.2-7.4$ for 24 hours, washed by cacodylate buffer $0.1 \mathrm{M}$, postfixed in $1 \%$ osmium tetroxide, dehydrated through a graded series of ethanol $(50 \%, 70 \%, 95 \%$ and $100 \%)$, cleaned in propylene oxide and finally embedded in an Araldite mixture. The ultrathin sections were stained by uranyl acetate and lead citrate. $^{25}$ The NiNPs were photographed by FEI Tecnai Spirit 10 TEM, while the ultrathin sections of kidney were photographed by the JEOL JEM 1400 TEM. The number of mitochondria in the proximal convoluted tubules (PT) cells per $100 \mu \mathrm{m}^{2}$ area of the electron micrographs were counted according to the method of Adachi $1967 .^{26}$

\section{Immunohistochemical analysis}

For detection of apoptotic cells, TUNEL assay was performed using the HRP-DAB (ab206386) kit. Immunohistochemical kits for the detection of $\mathrm{p} 53$ were used (manufactured by Leica Biosystems Newcastle Ltd, Newcastle upon Tyne, UK) and 
staining was carried out using an automated immunostainer (AutostainerLink48 DAKO, Agilent, Santa Clara, CA, USA).

\section{Blood collection and biochemical analysis}

Blood samples were collected from all anesthetized rats through cardiac puncture in which the collected blood samples were immediately placed into gel tubes for serum collection, then later centrifuged (Hettich D-78532/ Germany) at $3000 \mathrm{rpm}$ for 15 minutes. The sera were stored at $-80^{\circ} \mathrm{C}$ (Sanyo - Ultra - Low Temperature, Moriguchi, Osaka, Japan) for the measurement of creatinine. Serum creatinine, urea and uric acid were determined spectrophotometrically (Cobas c311 Hitachi, Tokyo, Japan) using a kit from BIOLABO S.A.S. (Maizy, France) at $500 \mathrm{~nm}$. Phosphorous was also estimated in serum using the ABNOVA calorimetric kits (Catalaog No. KA0815).

\section{Statistical analysis}

Statistical analyses were performed using Students $t$-test. Data were reported as mean \pm standard errors (SEs). $P$-values $\leq 0.05$ were considered significant.

\section{Results}

Throughout the study, the rats displayed no behavioral changes or unusual responses following treatment with NiNPs. Moreover, none of the rats died due to the treatment.

The shape and size of the $20 \mathrm{~nm}$ NiNPs are shown in Figure 1. Following the examination of the electron micrographs, the mean size of the NiNPs was found to be $19.84 \mathrm{~nm}$ \pm 3.01 (Figure 1B), which was approximately close to the size reported by the manufacturer.

The electron microscope images confirmed the internalization of the NiNPs into the kidney tissues of the treated rats, and were localized within lysosome-vacuoles in the epithelial cells lining PT (Figure 2A and B) or in the interstitial cells adherent to the basement membrane of the PT (Figure 2C and D). The NiNPs aggregated near the basement membrane appeared either as individual free particles or localized within vacuoles. The NiNPs were also found as individual particles in the cytoplasm of PT cells passed through the basement membrane (Figure 2D). Several numbers of NiNPs were also seen as individual particles within the brush border present in the lumen of the PT (Figure 2E).

As shown in Figure 3, NiNPs also accumulated in the interstitial region of the cells and close to the basement membrane of the PT. The administration of NiNPs caused alterations in structure of the lateral ridges and disturbed the structure of the basal microvilli in the treated rats compared to the control group (Figure 4). Subsequently, the damage that occurred in the kidneys following the exposure of the rats to NiNPs affected the level of serum creatinine, urea, uric acid and phosphorus compared to the control group (Table 1).

Within the glomerulus, NiNPs administration caused degeneration of podocytes and mesangial cells; the podocytes shrank and their nuclei condensed, while the mesangial cells displayed fragmented nuclei (Figure 5). Furthermore, the foot processes of the podocytes, which normally form a slit in between, appeared fused in some
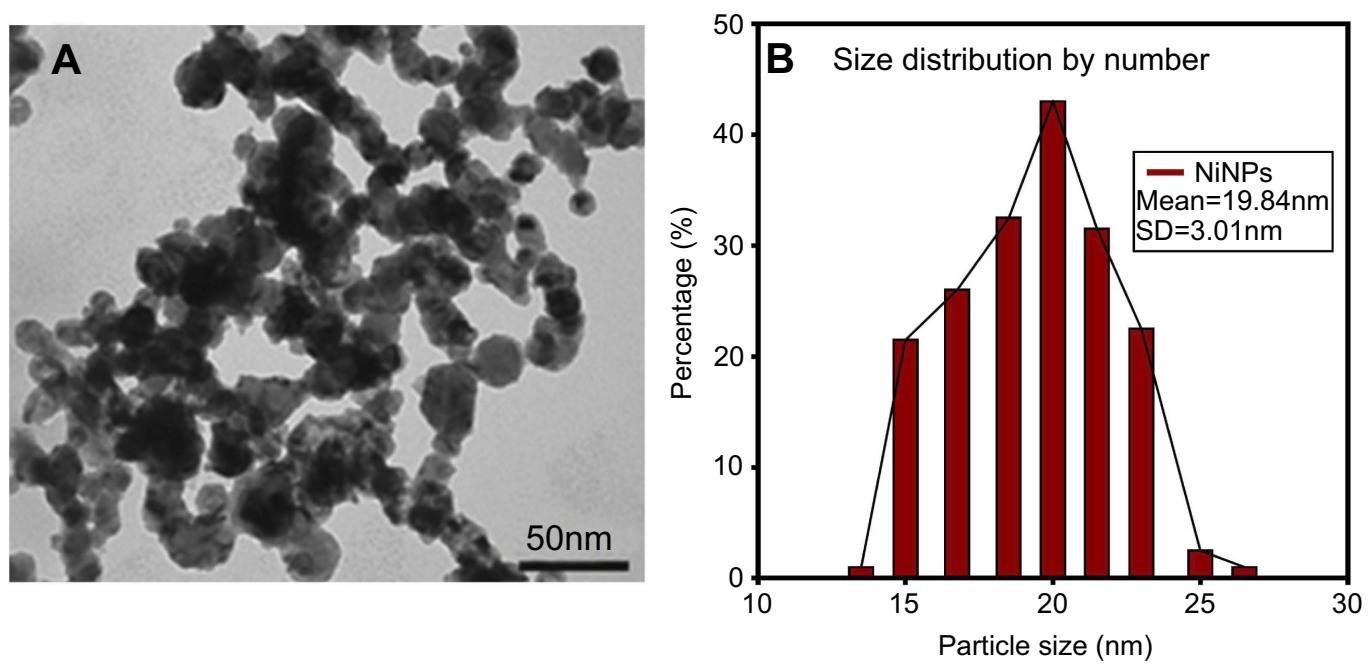

Figure I NiNPs shape and size: (A) the shape of NiNPs as revealed by transmission electron micrographs of NiNPs. (B) Size distribution by number using a large number of TEM photos.

Abbreviations: NiNPs, nickel nanoparticles; SD, standard deviation; TEM, transmission electron microscope. 


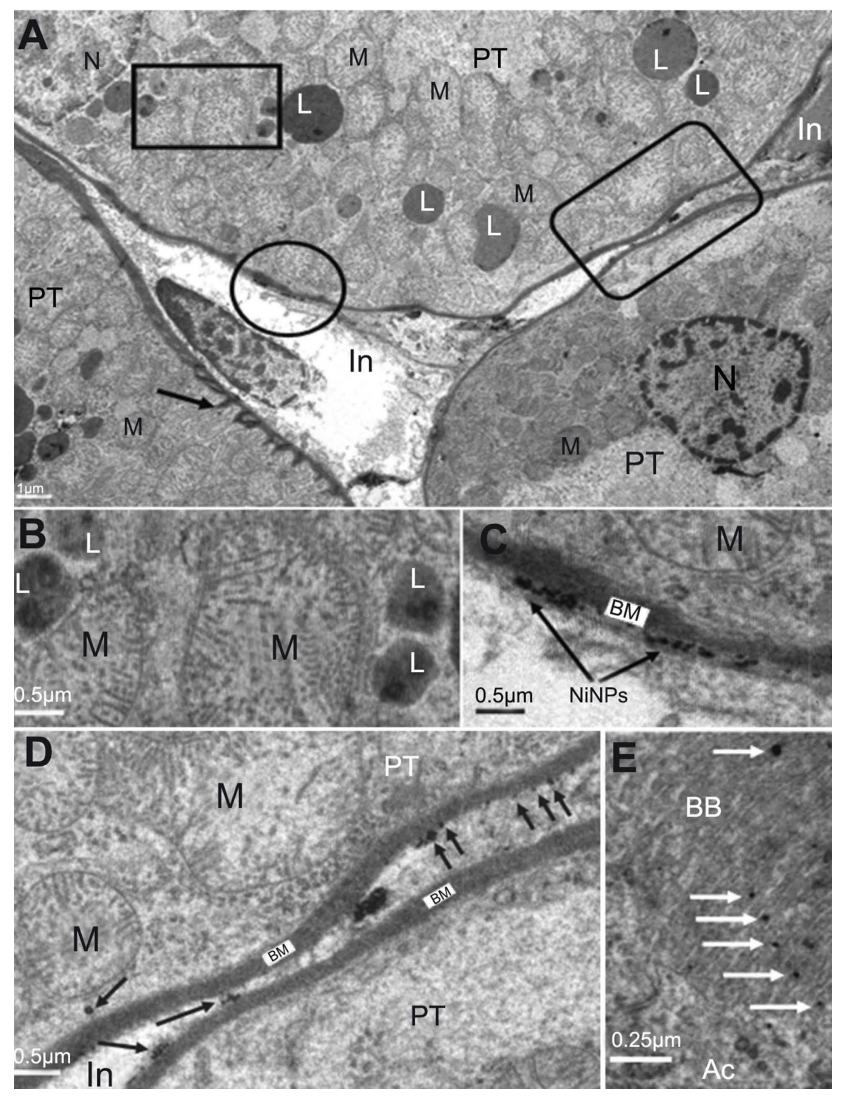

Figure 2 Internalization of NiNPs in rat kidney. (A) PT cell with different NiNPs localizations, within lysosome-like vacuoles (rectangle) and adhered to the basement membrane (circle and round curved rectangle) as shown in (B-D) respectively. Arrow indicates the lateral ridge of the basement membrane. (B) Lysosomelike vacuoles containing dense particles. (C) NiNPs (arrows) adhered to the basement membrane (BM). (D) NiNPs (arrows) either as individual particles inside the PT cell or adhered to the basement membrane of both adjacent PT cells. (E) Apical cytoplasmic region of PT cells showing discharged NiNPs (arrows) through the brush border into the lumen.

Abbreviations: PT, proximal convoluted tubule; NiNPs, nickel nanoparticles; BM, basement membrane; L, lysosome-like vacuoles; In, interstitial region; Ac, apical cytoplasmic region; $\mathrm{BB}$, brush border; $\mathrm{M}$, mitochondria; $\mathrm{N}$, nucleus.

sites forming a continuous layer (Figure 6A). The mode of cell death of mesangial cells appeared to be apoptotic, which was confirmed by a number of apoptotic bodies that were engulfed by adjacent neutrophil (Figure 6B). The mode of cell death in the glomerular cells was confirmed using the TUNEL assay and p53 immunohistochemical staining (Figure 7).

As clearly shown in Figure 8, exposure to NiNPs caused fusion or effacement of secondary foot processes of the podocytes. Both the proximal and distal renal tubules displayed degeneration of epithelial cells following NiNPs exposure, where some degenerated cells and cellular debris were shed into the lumen of the tubules (Figure 9).

The most prominent effects of the NiNPs on the appearance of the renal tubules, as ultrastructurally revealed, were the lower density and the structural damage of the mitochondria

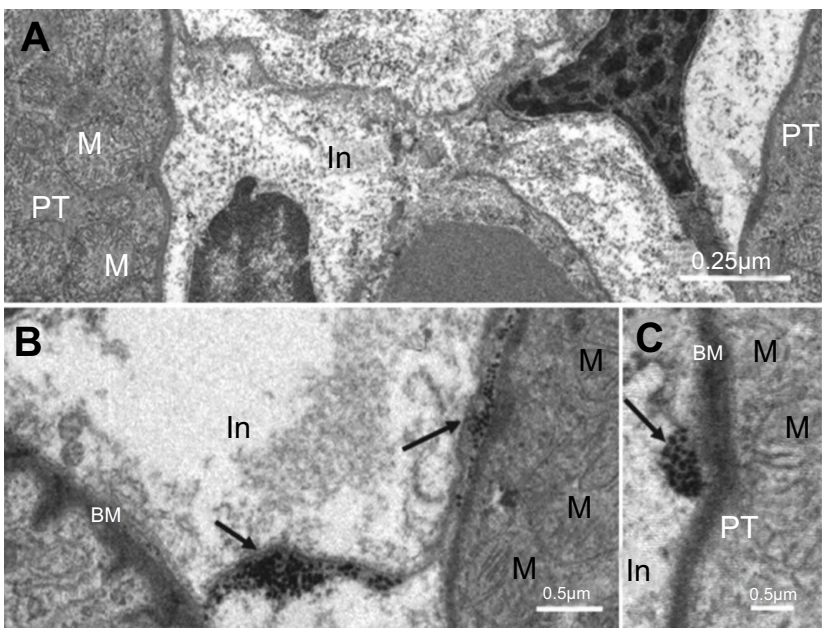

Figure 3 Internalization of NiNPs into the interstitial region of kidney cortex: (A) control group showing interstitial region between two proximal convoluted tubules. (B and $\mathbf{C}$ ) the NiNP-treated group showing (B) deposition of NiNPs close to membranes (arrows), and (C) number of electron dense particles within vacuole close to the PT basement membrane (arrow).

Abbreviations: PT, proximal convoluted tubule; NiNPs, nickel nanoparticles; BM, basement membrane; In, interstitial region; $M$, mitochondria.

compared to the control (Figure 10). Figure 10B shows NiNPs uptake by mitochondria and their transmembrane transport between the two adjacent PT cells through endocytosis. A significant decrease $(P \leq 0.05)$ occurred in the mitochondrial number in the PT cells of the NiNPs-treated rats compared to control (Figure 11). In comparison to the normal ultrastructural architecture of rough endoplasmic reticulum (RER)mitochondria close location system, a number of electrondense particles of variable size, probably NiNPs, were also found to be adherent to the region of the RER that attached to the mitochondria (Figure 12). NiNP-like particles were detected in the cells of the distal tubule, some were close to the plasma membrane, others were found as individual particles in the cytoplasm, and the rest of the particles were engulfed in vacuoles. Interestingly, a mitochondrion was found engulfing a particle-containing vacuole (see black box in Figure 13).

\section{Discussion}

Manufactured NPs and their applications are expanding in the industry, commodities, biology and medicine. This has led to an increased number of studies to improve our understanding regarding the entry of these NPs into different cell types in vivo in order to examine their effects on cellular responses. ${ }^{27-29}$

In this study, the cellular ultrastructural localization of NiNPs was found to be within vacuoles or were found as free individual particles or aggregated/accumulated particles 


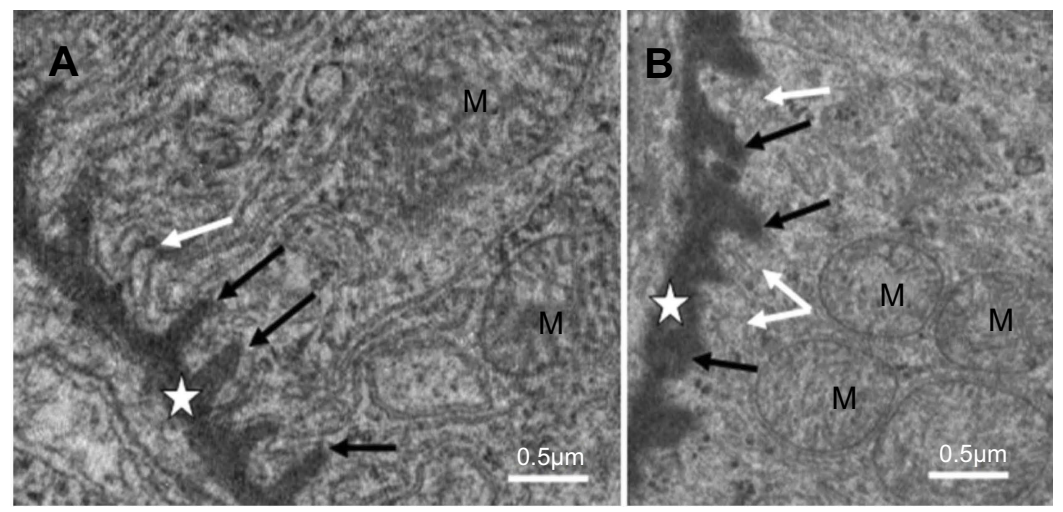

Figure 4 Basal region in proximal convoluted tubule cells of NiNP-treated rats. (A) Basal villi of control group (white arrow), lateral ridges (black arrows) extending from PT basement membrane (star). (B) Thickened basement membrane (star), disturbed basal villi (white arrows) and affected lateral ridges (black arrows).

Abbreviations: NiNPs, nickel nanoparticles; M, mitochondria; PT, proximal convoluted tubule.

Table I Biochemical analysis in the serum of NiNP-treated rats

\begin{tabular}{|l|l|l|}
\hline Parameters & Control & NiNPs \\
\hline Creatinine $(\mathrm{mg} / \mathrm{dL})$ & $0.46 \pm 0.03$ & $0.71 \pm 0.07^{*}$ \\
Urea $(\mathrm{mg} / \mathrm{dL})$ & $20.33 \pm 3.12$ & $31.22 \pm 4.56^{*}$ \\
Uric acid $(\mathrm{mg} / \mathrm{dL})$ & $17.33 \pm 2.12$ & $26.21 \pm 3.1 I^{*}$ \\
Phosphorus $(\mathrm{mg} / \mathrm{dL})$ & $1.18 \pm 0.42$ & $1.73 \pm 0.5 \mathrm{I}$ \\
\hline
\end{tabular}

Note: *Significant difference compared to control at $P \leq 0.05$. Data shown as mean $\pm \mathrm{SE}$. Abbreviation: NiNPs, nickel nanoparticles.

in renal tubule epithelial cells, and in the interstitium close to the basement membrane of the cells lining the renal tubules. The accumulation of NiNPs at the PT membrane may be due to many factors; firstly, difficulty in their indirect penetration rate leading to accumulation, secondly: due to their high extracellular concentration, ${ }^{30,31}$ the repetitive daily injections, thirdly: due to their large size. Moreover, physicochemical properties of the NPs could be another possible factor behind their aggregation, accumulation, and adhesion. ${ }^{31-33}$ Previously, nickel was found to accumulate in the lysosomes of proximal tubule cells and this was attributed to their strongly acidic, lipid-like properties that allow them to bind cationic and lipophilic substances. ${ }^{34}$ Herein, NiNPs penetrated the basement membrane of the PT cells after their adhesion to the plasma membrane, but the penetration rate was slow although there was a high density of accumulated particles. Although lysosome-like vacuoles contained electron-dense particles, endocytosis of NiNPs close to the basement membrane couldn't be observed in this study. Endocytosis, on the other hand, was detected at the plasma membrane of adjacent PT cells. Nanoparticles such as titanium dioxide $\left(\mathrm{TiO}_{2} \mathrm{NPs}\right)$, gold (AuNPs), silicon dioxide $\left(\mathrm{SiO}_{2} \mathrm{NPs}\right)$, iron oxide $\left(\mathrm{Fe}_{3} \mathrm{O}_{4}\right.$ $\mathrm{NPs}$ ) and silver (AgNPs) have been found to penetrate directly into the plasma membrane. ${ }^{1}$ Modes of direct penetration include diffusion, permeation, pore formation ${ }^{35}$ and wrapping by membrane. ${ }^{5}$ Although free individual NiNPs are detected close to the basement membrane, the mode of direct penetration couldn't be confirmed, but the most probable pathway may be through translocation. ${ }^{36}$ The size of the NiNPs
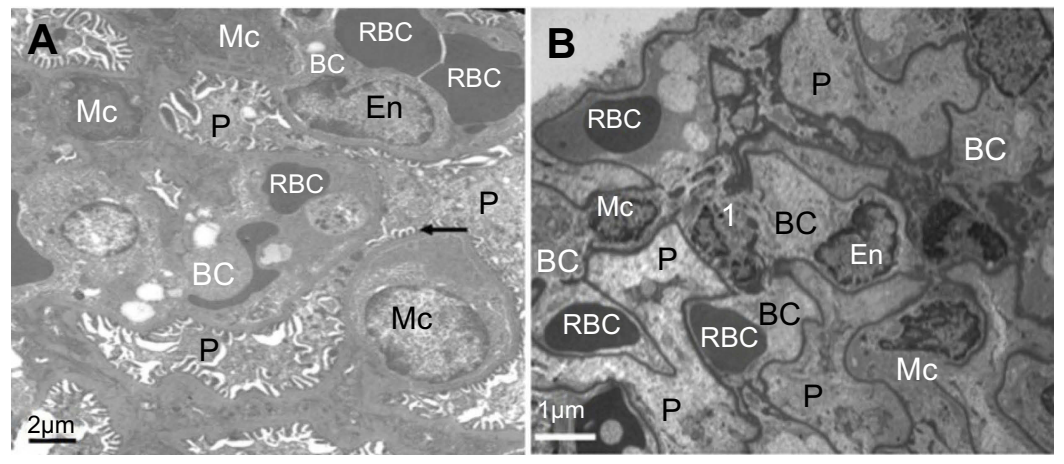

Figure 5 Ultrastructure of glomerulus after NiNPs administration: (A) control group showing well developed podocytes with their foot processes (arrow), endothelial and mesangial cells. Red blood cells in the blood capillaries are seen; (B) the NiNP-treated group showing degenerated podocytes and mesangial cells. The nuclei of mesangial cells (I) are seen fragmented.

Abbreviations: P, podocytes; EN, endothelial cells; RBC, red blood cells; BC, blood capillary; Mc, mesangial cells; NiNPs, nickel nanoparticles. 

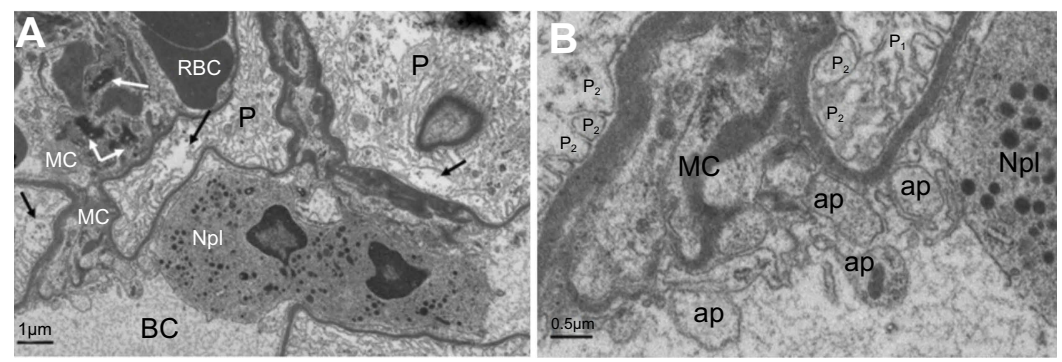

Figure 6 Ultrastructure changes in podocytes and mesangial cells in NiNP-treated rat kidney. (A) Degeneration of podocytes showing the fusion of foot processes (effacement) (arrows). Mesangial cells appeared degenerated having fragmented nucleus (black arrows). Neutrophil in the blood capillary showing phagocytosis of the degenerating mesangial cells. (B) Enlargement of (A) showing the phagocytosis process and engulfing of apoptotic bodies of mesangial cells and fusion of foot processes of podocytes.

Note: $P_{1}$, primary podocyte foot processes; $P_{2}$, secondary podocyte foot processes.

Abbreviations: NiNPs, nickel nanoparticles; P, podocytes; RBC, red blood cells; BC, blood capillary; MC, mesangial cells; Npl, neutrophil; ap, apoptotic bodies.

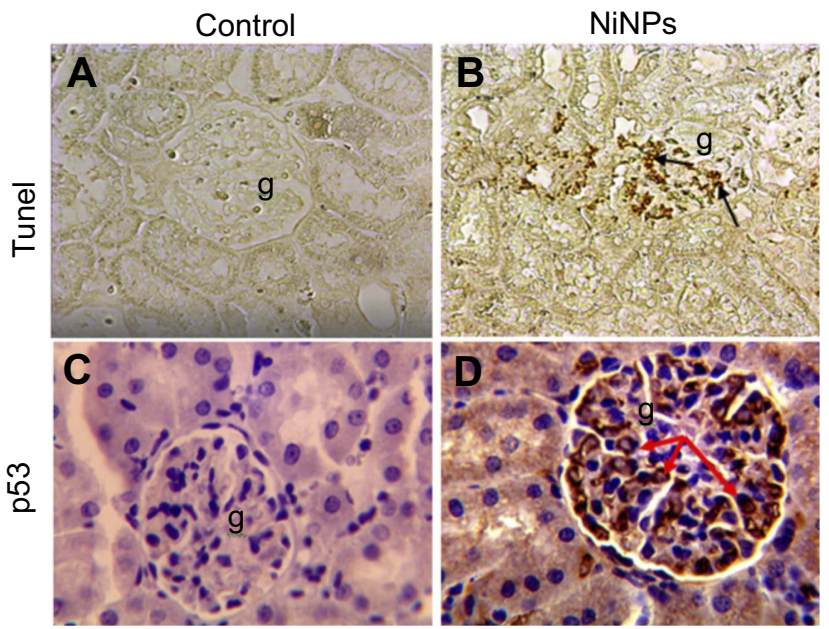

Figure 7 Immunohistochemical images of apoptotic cells in the glomerulus of NiNP-treated rat kidney. (A) Control group showing negative Tunel reaction. (B) NiNPs group showing positive Tunel reaction (arrows). (C) Control group showing no positive $\mathrm{p} 53$ reaction. (D) NiNPs group showing positive $\mathrm{p} 53$ reaction (arrows). Abbreviations: NiNPs:, nickel nanoparticles; g, glomerulus.

facilitated their entrance into the target cells. ${ }^{1} \mathrm{NP}$ size, shape and core composition are strong determinants of cellular uptake and accumulation. ${ }^{36,37}$ Although it is still unclear whether translocation, distribution, and elimination are affected by NP size, ${ }^{38}$ the size of NPs remains a critical factor in their internalization and accumulation. The size of NiNPs used in this study was $20 \mathrm{~nm}$ in diameter; this could facilitate NiNPs entrance into cells. ${ }^{1}$ Nevertheless, NPs with a size ranged of $20-50 \mathrm{~nm}$ are considered appropriate application involving drug delivery. ${ }^{39} \mathrm{NPs}$ with a size less than $20 \mathrm{~nm}$ (eg $10 \mathrm{~nm}$ ) have been shown to accumulate in the liver and spleen of rats, ${ }^{40}$ while NPs of larger size $(20-100 \mathrm{~nm})$ have been found to accumulate in the kidney. ${ }^{41}$ On the other hand, NPs with a size less than $20 \mathrm{~nm}$ could be rapidly cleared by the kidneys, ${ }^{42,43}$ while larger NiNPs may be difficult to be uptaken by cells. ${ }^{44}$ The rapid clearance of NPs limits their use because

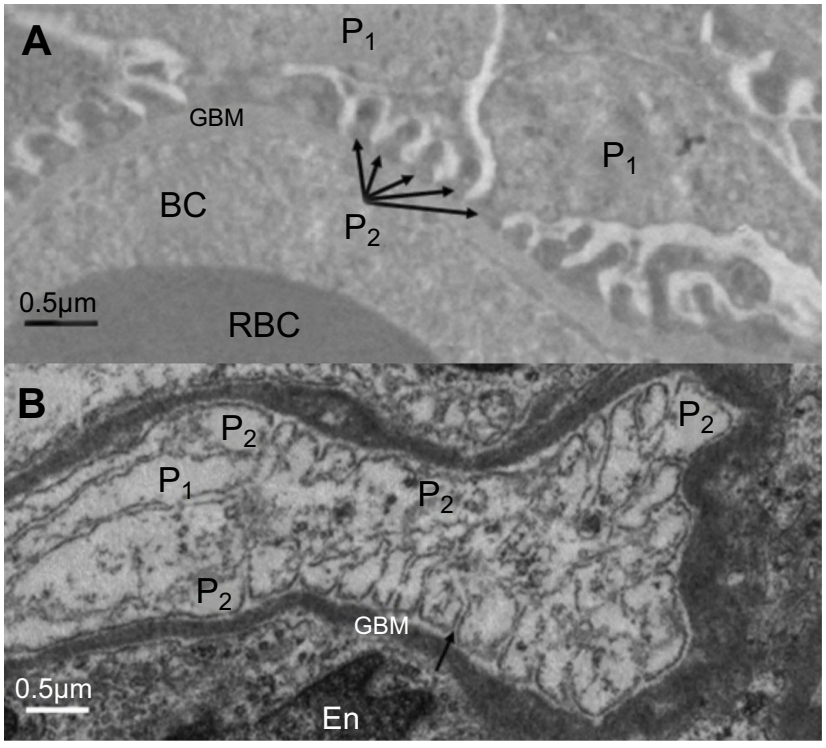

Figure 8 Ultrastructure changes in the podocytes foot processes. (A) Control group showing regular slit distances due to normal secondary foot processes structure (arrows; $P_{2}$ ). (B) Fusion (effacement) of some of the secondary foot processes $\left(P_{2}\right)$. Arrow indicates a slit.

Note: $P_{1}$, primary podocyte foot processes; $P_{2}$, secondary podocyte foot processes. Abbreviations: En, endothelial cells; RBC, red blood cells; BC, blood capillary; NiNPs, nickel nanoparticles; GBM, glomerular basement membrane.

they will take longer to reach target tissues. ${ }^{45}$ Currently, no agreement has been reached on the effect of size on the cellular uptake and accumulation of NPs. For example, Cho et al ${ }^{46}$ observed maximum cellular uptake for AuNPs with a size less than $30 \mathrm{~nm},{ }^{46}$ while Chithrani and $\mathrm{Chan}^{47}$ found maximum uptake for AuNPs with a size between 50 and $70 \mathrm{~nm} .{ }^{47}$ These discrepancies may be due to the varied physicochemical properties of NPs. ${ }^{37}$ The size and surface charge are found to interact in an interrelated fashion to modulate nanoparticle uptake into cells. For anionic AuNPs, cellular uptake is decreased with increasing size, whereas with cationic particles, cell uptake is increased with increasing particle size. ${ }^{48}$ In addition to cellular uptake, particle size can also affect the 

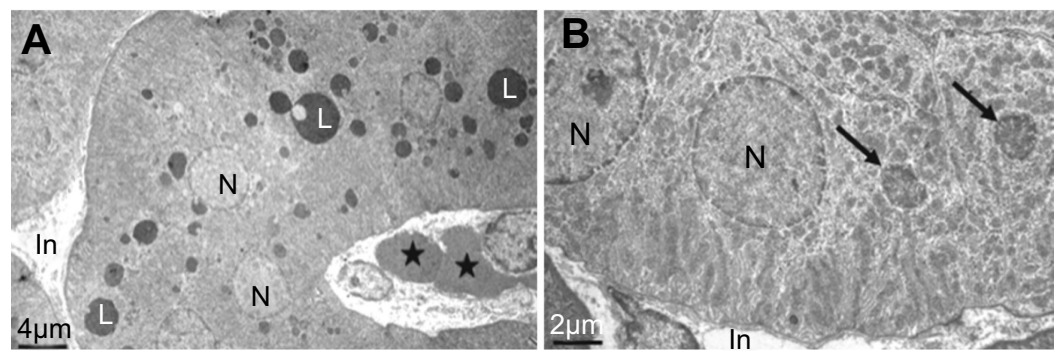

Figure 9 Ultrastructure of renal tubules of kidney of rats treated with NiNPs. (A) Proximal convoluted tubules of the NiNP-treated group showing variable sized lysosome-like vacuoles. Cellular debris is seen in the lumen of the duct shed from the renal tubules (stars). (B) Distal tubule of NiNP-treated rat showing pyknotic nuclei (arrows) against normal nuclei. Abbreviations: NiNPs, nickel nanoparticles; L, lysosome-like vacuoles; N, nucleus; In, interstitial region.
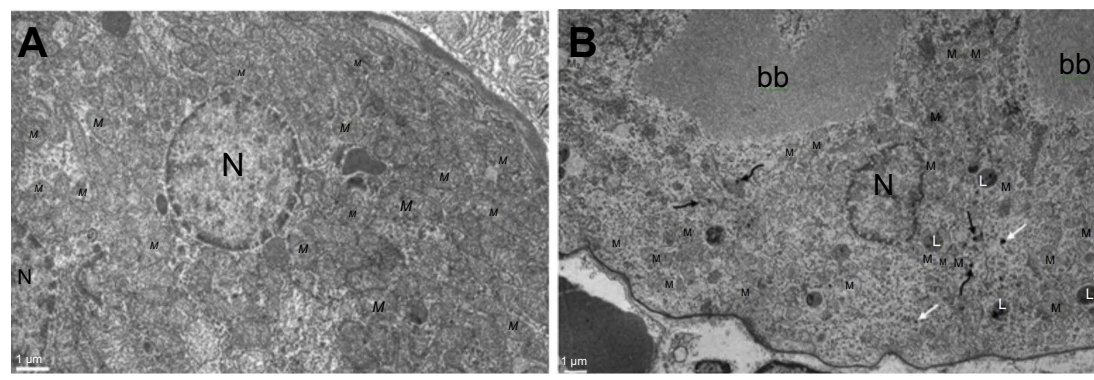

Figure 10 Electron microscope images of proximal convoluted tubule showing the status of mitochondria. (A) In the control group showing the normal density and ultrastructure. (B) In NiNP-treated rats showing the low density normal and damaged mitochondria. NiNPs are seen in different locations, free in the cytoplasm (black arrow), endocytosed by plasma membrane (white arrow), within lysosome-like vacuoles and uptaken by mitochondria (curved arrow connector).

Note: $M_{2}$, damaged mitochondria.

Abbreviations: NiNPs, nickel nanoparticles; PT, proximal convoluted tubule; L, lysosome-like vacuoles; N, nucleus; M, mitochondria; bb, brush border.

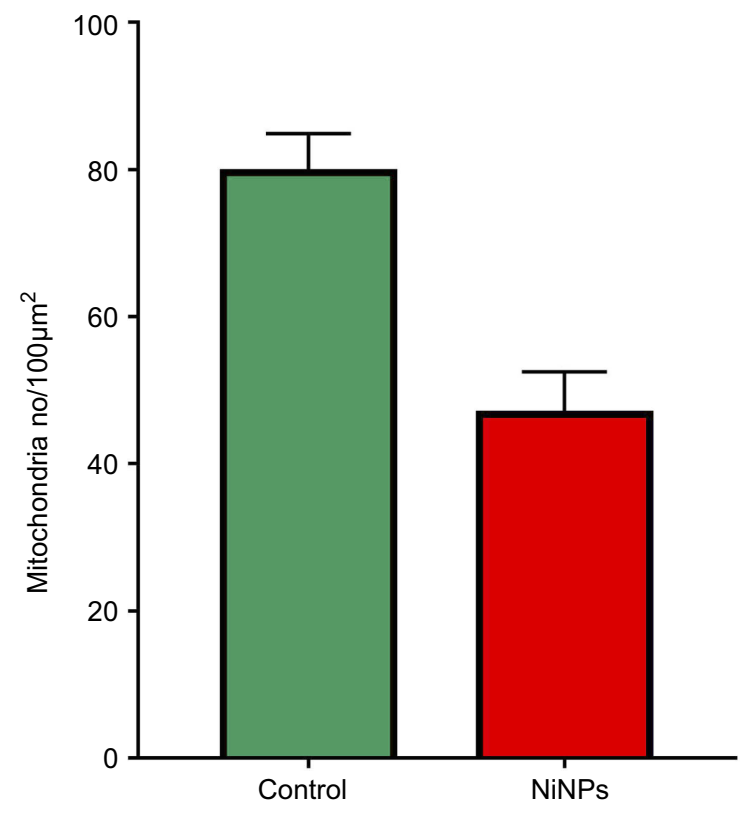

Figure I I Mitochondria number counted by TEM photomicrographs per $100 \mu \mathrm{m}^{2}$ area in PT cells of NiNPs treated rats which showed a significant decrease compared to control.

Note: *Significant decrease at $P \leq 0.05$.

Abbreviation: NiNPs: nickel nanoparticles; TEM, transmission electron microscope; PT, proximal convoluted tubule. mode of endocytosis. ${ }^{49}$ With respect to size-dependent toxicity, small-sized NPs have larger surface area and particle concentrations than NPs of larger size which may increase the chance of interaction with surrounding macromolecules such as DNA and proteins, and as a consequence, trigger adverse responses. ${ }^{50}$ Smaller-sized NPs have been shown to induce much higher production of ROS and interleukin- 8 than NPs of larger size. ${ }^{51,52}$

On the other hand, the route of administration may play a significant role in NPs accumulation in different organs causing different levels of toxicity. In the present investigation, the intraperitoneal route of administration was selected since it is widely used in NPs research, particularly in nanomedicine. ${ }^{53}$ It is well known that drug absorption via the intraperitoneal route is good and rapid due to the high intensity of blood vessels and lymph in the murine peritoneum. ${ }^{54}$ Intraperitoneal injection has been demonstrated to improve the uptake of NP-labeled macromolecules compared with the intravenous route. ${ }^{55}$ Intraperitoneal injection may cause a higher accumulation of AuNPs in kidneys compared with micromolecules. ${ }^{56}$ 

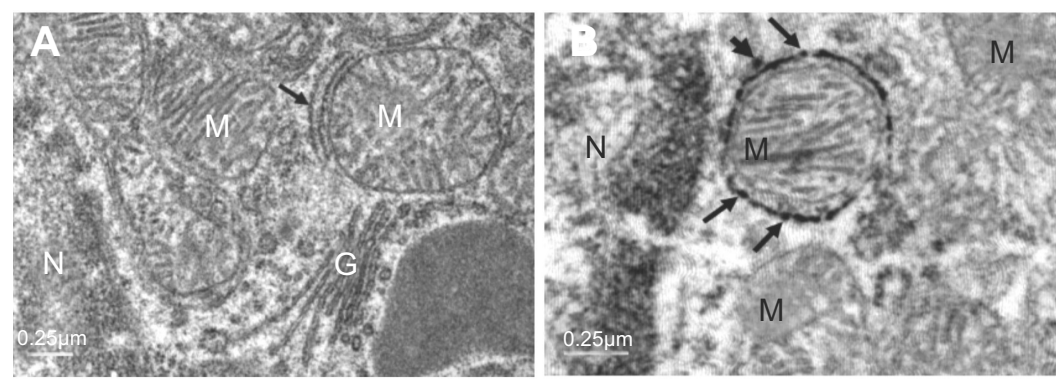

Figure 12 NiNPs intracellular localization in PT cells: (A) control group with normal ultrastructure of mitochondria, RER (arrow) and Golgi apparatus. (B) The NiNPtreated group showing electron dense particles, probably NiNPs, adhered to the RER which surround the mitochondria (arrows) and some are still free (arrow head). Abbreviations: NiNPs, nickel nanoparticles; N, nucleus; RER, rough endoplasmic reticulum; M, mitochondria; PT, proximal convoluted tubule; G, Golgi apparatus.

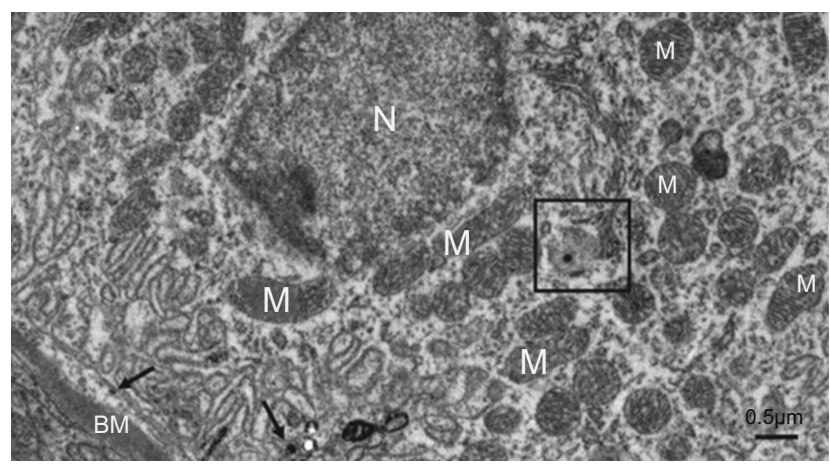

Figure 13 A distal tubule lining epithelial cell in the NiNP-treated group. Few numbers of mitochondria are seen. The box shows a vacuole containing an electron dense particle and whole vacuole is seen surrounded by an adjacent mitochondrion. Number of electron dense particles, probably NiNPs, near the basement membrane are seen (arrows).

Abbreviations: NiNPs, nickel nanoparticles; $M$, mitochondria; $N$, nucleus; $B M$, basement membrane.

As revealed by the TEM images, the NiNPs used here were spherical. Previously, when a comparison between cubic, spherical and rod-like gold nanoparticles was made, spherical particles showed the highest cellular uptake. ${ }^{57}$ During renal excretion, the spherical NPs are cleared faster than rod NPs. ${ }^{58}$ Differential toxicity due to the varying shapes of NPs has been reported. For example, the dendritic structure of NiNPs is found to result in higher toxicity in comparison to a spherical structure. ${ }^{59}$ In a toxicity study using AgNPs with epithelial cells, wireshaped silver was found to be more toxic than sphereshaped silver. ${ }^{60}$ Localization of clusters of NiNPs inside vacuoles in our images may be due to their agglomeration through curvature- mediated attraction. ${ }^{61}$

Biochemically, sub-acute administration of NiNPs caused a significant increase of serum creatinine, urea and uric acid and a non significant increase of phosphorous level compared to the control. Recently, acute administration of nickel oxide nanoparticles (NiONPs) was found to induce an elevation of creatinine level. ${ }^{62}$ The high levels of creatinine may reflect an abnormal glomerular filtration rate due to metals accumulation in kidneys. ${ }^{63}$ Degeneration of renal tubular cells and mitochondrial damage due to NiNPs localization and accumulation, as revealed by this present work, may be related to these biochemical changes. ${ }^{64}$

In this study, NiNPs caused damage to the mitochondria which also displayed a lower density. Several studies suggested the targeting of mitochondria by NPs due to their ability to bind with mitochondrial complex II of the electron transport chain to induce apoptosis. ${ }^{33,65}$ Mitochondria-targeting NPs have become a potential novel strategy in drug delivery for cancer therapy. ${ }^{65,66}$ The NiNPs-induced damage of the mitochondrial inner membrane, as revealed by the current investigation and consistently observed in previous work, may result in cytochrome c release leading to apoptosis. ${ }^{66,67}$ NiNPs uptake by mitochondria may be behind elevated oxidative stress, since mitochondria play a crucial role in cellular oxidative stress. ${ }^{68,69}$ Recently, other NPs such as silica NPs, were found to induce oxidative stress causing impaired mitochondrial function leading to apoptosis. ${ }^{67}$ NiNPs-induced oxidative stress has been suggested to cause nanotoxicity, particularly induction of apoptosis. ${ }^{7}$ The observation of NiNPs in the apical villi of PT cells in the present study indicates that excretion of NiNPs may occur via the urinary route, but further confirmation is required through further NP elimination studies. ${ }^{70}$

\section{Conclusion}

NiNPs were found to induce various cellular ultrastructure changes in the kidneys of rats. The cellular ultrastructure alterations, which subsequently affected renal function may be due to the direct contact of NiNPs with the renal cells or due to the oxidative stress induced by NiNPs. The NiNPs used different ways to translocate into the cytoplasm of the PT cells across the basement membrane and 
through the plasma membrane of the two adjacent PT cells. Such pathways of internalization may be affected by the characteristic physicochemical properties of the NPs.

\section{Acknowledgments}

We thank Prof. S. Polat in Cukurova University, Adana, Turkey; Dr Dara K. Mohammad, Karolinska Institutet, Solna, Sweden; Prof. Mojtaba Falahati, Azadi Islamic University, Tehran, Iran; and Dr Abbas Salihi, Salahaddin University-Erbil, Erbil, Iraq for their technical assistance. The authors also acknowledge Dr Fawaz Abumaray, Karolinska Institutet, Solna, Sweden for the linguistic revision.

\section{Disclosure}

The authors report no conflicts of interest in this work.

\section{References}

1. Nakamura H, Watano S. Direct permeation of nanoparticles across cell membrane: a review. KONA Powder Particle J. 2018;35:49-65.

2. Savolainen K, Alenius H, Norppa H, Pylkkanen L, Tuomi T, Kasper G. Risk assessment of engineered nanomaterials and nanotechnologies-a review. Toxicology. 2010;269:92-104. doi:10.1016/j.tox.2010.01.013

3. Khan I, Saeed K, Khan I. Nanoparticles: properties, applications and toxicities. Arabian J Chem. 2017. doi:10.1016/j.arabjc.2017.05.011

4. Chen P, Zhang Z, Xing J, Gu N, Ji M. Physicochemical properties of nanoparticles affect translocation across pulmonary surfactant monolayer. Mol Phys. 2017;115:3143-3154. doi:10.1080/ 00268976.2017 .1351005

5. Nakamura H. Molecular dynamics simulation study on interaction of nanoparticle with cell membrane. Japanese Journal of Multiphase Flow. 2018;32(3):321-328.

6. Kong L, Hu W, Lu C, Cheng K, Tang M. Mechanisms underlying nickel nanoparticle induced reproductive toxicity and chemo-protective effects of vitamin C in male rats. Chemosphere. 2019;218:259-265. doi:10.1016/j.chemosphere.2018.11.128

7. Yu S, Liu F, Wang C, et al. Role of oxidative stress in liver toxicity induced by nickel oxide nanoparticles in rats. Mol Med Rep. 2018;17:3133-3139. doi:10.3892/mmr.2017.8226

8. Latvala S, Hedberg J, Di Bucchianico S, et al. Nickel release, ROS generation and toxicity of $\mathrm{Ni}$ and $\mathrm{NiO}$ micro- and nanoparticles. PLoS One. 2016;11:e0159684. doi:10.1371/journal.pone.0159684

9. Tahereh Razavipour S, Behnammorshedi M, Razavipour R, Ajdary M. The Toxic Effect of Nickel Nanoparticles on Oxidative Stress and Inflammatory Markers. Biomedical Research. 2015;26(2):370-374.

10. Ma C, Song M, Zhang Y, Yan M, Zhang M, Bi H. Nickel nanowires induce cell cycle arrest and apoptosis by generation of reactive oxygen species in HeLa cells. Toxicol Rep. 2014;1:114-121. doi:10.1016/j.toxrep.2014.04.008

11. Meng H, Xia T, George S, Nel AE. A predictive toxicological paradigm for the safety assessment of nanomaterials. ACS Nano 2009;3:1620-1627. doi:10.1021/nn9005973

12. Nel A, Xia T, Madler L, Li N. Toxic potential of materials at the nanolevel. Science. 2006;311:622-627. doi:10.1126/science.1114397

13. Zhang Q, Kusaka Y, Sato K, Nakakuki K, Kohyama N, Donaldson K. Differences in the extent of inflammation caused by intratracheal exposure to three ultrafine metals: role of free radicals. $J$ Toxicol Environ Health A. 1998;53:423-438.
14. Abudayyak M, Guzel E, Ozhan G. Nickel oxide nanoparticles induce oxidative DNA damage and apoptosis in kidney cell line (NRK-52E). Biol Trace Elem Res. 2017;178:98-104. doi:10.1007/s12011-016-0892-z

15. Saquib Q, Attia SM, Ansari SM, et al. p53, MAPKAPK-2 and caspases regulate nickel oxide nanoparticles induce cell death and cytogenetic anomalies in rats. Int $J$ Biol Macromol. 2017;105:228-237. doi:10.1016/j.ijbiomac.2017.07.032

16. Amara S, Slama IB, Mrad I, et al. Effects of zinc oxide nanoparticles and/or zinc chloride on biochemical parameters and mineral levels in rat liver and kidney. Hum Exp Toxicol. 2014;33:1150-1157. doi:10.1177/0960327113510327

17. Alidadi H, Khorsandi L, Shirani M. Effects of Quercetin on Tubular Cell Apoptosis and Kidney Damage in Rats Induced by Titanium Dioxide Nanoparticles. The Malaysian Journal of Medical Sciences. 2018;25(2):72-81.

18. Abdel-Wahhab MA, Aljawish A, El-Nekeety AA, Abdel-Aziem SH, Hassan NS. Chitosan nanoparticles plus quercetin suppress the oxidative stress, modulate DNA fragmentation and gene expression in the kidney of rats fed ochratoxin A-contaminated diet. Food Chem Toxicol. 2017;99:209-221. doi:10.1016/j.fct.2016.12.002

19. Mohammadi Fartkhooni F, Noori A, Mohammadi A. Effects of Titanium Dioxide Nanoparticles Toxicity on the Kidney of Male Rats. International Journal of Life Sciences. 2016;10(1):65-69.

20. Amudha K, Pari L. Beneficial role of naringin, a flavanoid on nickel induced nephrotoxicity in rats. Chem Biol Interact. 2011;193:57-64. doi:10.1016/j.cbi.2011.05.003

21. National Research Council Committee for the Update of the Guide for the CUoL, Animals. The National Academies Collection: Reports Funded by National Institutes of Health. Guide for the Care and Use of Laboratory Animals. Washington, DC: National Academies Press (US), National Academy of Sciences; 2011.

22. Andrews K. UBC Animal Care Guidelines. Acc-2012-Tech10. Canada: University of British Columbia; 2014.

23. Magaye RR, Yue X, Zou B, et al. Acute toxicity of nickel nanoparticles in rats after intravenous injection. Int $J$ Nanomedicine. 2014;9:1393-1402. doi:10.2147/IJN.S56212

24. Abdulqadir S, Aziz F. Nephrotoxicity of Nickel Nanoparticles in Rat: Effect of Different Doses. Presented at: 10th International Conference on Chemical, Biological, Environmental and Medical Sciences. March 21-22; 2018; Istanbul, Turkey.

25. Kiernan J. Histological and Histochemical Methods: Theory and Practice. 5th ed. Banbury: Scion Publishing Ltd; 2015.

26. Adachi F. Electron microscopic investigation of the regenerating hepatic cells of the senile rat. Nagoya $J$ Med Sci. 1967;30:109-120.

27. Foroozandeh P, Aziz AA. Insight into cellular uptake and intracellular trafficking of nanoparticles. Nanoscale Res Lett. 2018;13:339.

28. Panzarini E, Mariano S, Carata E, Mura F, Rossi M, Dini L. Intracellular transport of silver and gold nanoparticles and biological responses: an update. Int J Mol Sci. 2018;19(5):E1305.

29. Oh N, Park JH. Endocytosis and exocytosis of nanoparticles in mammalian cells. Int $J$ Nanomedicine. 2014;9(Suppl 1):51-63. doi:10.2147/IJN.S26592

30. Lawrence MG, Altenburg MK, Sanford R, et al. Permeation of macromolecules into the renal glomerular basement membrane and capture by the tubules. Proc Natl Acad Sci USA. 2017;114:2958-2963. doi:10.1073/pnas.1616457114

31. Shin SW, Song IH, Um SH. Role of physicochemical properties in nanoparticle toxicity. Nanomaterials. 2015;5:1351-1365. doi:10.3390/ nano5031351

32. Wu T, Tang M. Review of the effects of manufactured nanoparticles on mammalian target organs. J Appl Toxicol. 2018;38:25-40. doi: $10.1002 /$ jat.3499

33. Mallick A, More P, Syed MM, Basu S. Nanoparticle-mediated mitochondrial damage induces apoptosis in cancer. ACS Appl Mater Interfaces. 2016;8:13218-13231. doi:10.1021/acsami.6b00263 
34. Dingle JT, Barrett AJ. Uptake of biologically active substances by lysosomes. Proc $R$ Soc Lond B Biol Sci. 1969;173:85-93. doi:10.1098/rspb.1969.0040

35. Guarnieri D, Sabella S, Muscetti O, et al. Transport across the cell-membrane dictates nanoparticle fate and toxicity: a new paradigm in nanotoxicology. Nanoscale. 2014;6:10264-10273. doi:10.1039/c4nr02008a

36. Zhang S, Gao H, Bao G. Physical principles of nanoparticle cellular endocytosis. ACS Nano. 2015;9:8655-8671. doi:10.1021/ acsnano. 5 b03184

37. Wong AC, Wright DW. Size-dependent cellular uptake of DNA functionalized gold nanoparticles. Small. 2016;12:5592-5600. doi:10.1002/smll.201601697

38. Yang L, Kuang H, Zhang W, Aguilar ZP, Wei H, Xu H. Comparisons of the biodistribution and toxicological examinations after repeated intravenous administration of silver and gold nanoparticles in mice. Sci Rep. 2017;7:3303. doi:10.1038/s41598-017-03015-1

39. Ibrahim KE, Al-Mutary MG, Bakhiet AO, Khan HA. Histopathology of the liver, kidney, and spleen of mice exposed to gold nanoparticles. Molecules. 2018;23(8):E1848.

40. Ruiz A, Mancebo A, Beola L, Sosa I, Gutiérrez L. Doseresponse bioconversion and toxicity analysis of magnetite nanoparticles. IEEE Magn Lett. 2016;7:1-5. doi:10.1109/ LMAG.2016.2535414

41. Takeuchi I, Nobata S, Oiri N, Tomoda K, Makino K. Biodistribution and excretion of colloidal gold nanoparticles after intravenous injection: effects of particle size. Biomed Mater Eng. 2017;28:315-323. doi:10.3233/BME-171677

42. Sadat S, Jahan S, Haddadi A. Effects of Size and Surface Charge of Polymeric Nanoparticles on in Vitro and in Vivo Applications. Journal of Biomaterials and Nanobiotechnology. 2016;7:91-108.

43. Hoshyar N, Gray S, Han H, Bao G. The effect of nanoparticle size on in vivo pharmacokinetics and cellular interaction. Nanomedicine (Lond). 2016;11:673-692. doi:10.2217/nnm.16.5

44. Mao BH, Tsai JC, Chen CW, Yan SJ, Wang YJ. Mechanisms of silver nanoparticle-induced toxicity and important role of autophagy. Nanotoxicology. 2016;10:1021-1040. doi:10.1080/ 17435390.2016 .1189614

45. Sun YN, Wang CD, Zhang XM, Ren L, Tian XH. Shape dependence of gold nanoparticles on in vivo acute toxicological effects and biodistribution. J Nanosci Nanotechnol. 2011;11:1210-1216.

46. Cho EC, Au L, Zhang Q, Xia Y. The effects of size, shape, and surface functional group of gold nanostructures on their adsorption and internalization by cells. Small. 2010;6:517-522. doi:10.1002/ smll.200901622

47. Chithrani BD, Chan WC. Elucidating the mechanism of cellular uptake and removal of protein-coated gold nanoparticles of different sizes and shapes. Nano Lett. 2007;7:1542-1550. doi:10.1021/ n1070363y

48. Jiang W, Kim BYS, Rutka JT, Chan WCW. Nanoparticle-mediated cellular response is size-dependent. Nat Nanotechnol. 2008;3:145. doi:10.1038/nnano.2008.30

49. Liu X, Huang N, Li H, Jin Q, Ji J. Surface and size effects on cell interaction of gold nanoparticles with both phagocytic and nonphagocytic cells. Langmuir. 2013;29:9138-9148. doi:10.1021/ la401556k

50. Cho YM, Mizuta Y, Akagi JI, Toyoda T, Sone M, Ogawa K. Sizedependent acute toxicity of silver nanoparticles in mice. $J$ Toxicol Pathol. 2018;31:73-80. doi:10.1293/tox.2017-0043

51. Park J, Lim DH, Lim HJ, et al. Size dependent macrophage responses and toxicological effects of $\mathrm{Ag}$ nanoparticles. Chem Commun (Camb). 2011;47:4382-4384. doi:10.1039/c1cc10357a

52. Lopez-Chaves C, Soto-Alvaredo J, Montes-Bayon M, Bettmer J, Llopis J, Sanchez-Gonzalez C. Gold nanoparticles: distribution, bioaccumulation and toxicity. In vitro and in vivo studies. Nanomedicine. 2018;14:1-12. doi:10.1016/j.nano.2017.08.011
53. Almansour M, Alarifi S, Jarrar B. In vivo investigation on the chronic hepatotoxicity induced by intraperitoneal administration of $10-\mathrm{nm}$ silicon dioxide nanoparticles. Int J Nanomedicine. 2018;13:2685-2696. doi:10.2147/IJN.S162847

54. Zhang XD, Wu HY, Wu D, et al. Toxicologic effects of gold nanoparticles in vivo by different administration routes. Int $J$ Nanomedicine. 2010;5:771-781. doi:10.2147/IJN.S8428

55. Jung C, Kaul MG, Bruns OT, et al. Intraperitoneal injection improves the uptake of nanoparticle-labeled high-density lipoprotein to atherosclerotic plaques compared with intravenous injection: a multimodal imaging study in ApoE knockout mice. Circ Cardiovasc Imaging. 2014;7:303-311. doi:10.1161/CIRCIMAGING.113.000607

56. Abdelhalim MA. Uptake of gold nanoparticles in several rat organs after intraperitoneal administration in vivo: a fluorescence study. Biomed Res Int. 2013;2013:353695. doi:10.1155/2013/353695

57. Niikura K, Matsunaga T, Suzuki T, et al. Gold nanoparticles as a vaccine platform: influence of size and shape on immunological responses in vitro and in vivo. ACS Nano. 2013;7:3926-3938. doi:10.1021/nn3057005

58. Zhao Y, Wang Y, Ran F, et al. A comparison between sphere and rod nanoparticles regarding their in vivo biological behavior and pharmacokinetics. Sci Rep. 2017;7:4131. doi:10.1038/s41598-01703834-2

59. Ispas C, Andreescu D, Patel A, Goia DV, Andreescu S, Wallace KN. Toxicity and developmental defects of different sizes and shape nickel nanoparticles in zebrafish. Environ Sci Technol. 2009;43:6349-6356.

60. Stoehr LC, Gonzalez E, Stampfl A, et al. Shape matters: effects of silver nanospheres and wires on human alveolar epithelial cells. Part Fibre Toxicol. 2011;8:36. doi:10.1186/1743-8977-8-36

61. Reynwar BJ, Illya G, Harmandaris VA, Muller MM, Kremer K, Deserno M. Aggregation and vesiculation of membrane proteins by curvature-mediated interactions. Nature. 2007;447:461-464. doi:10.1038/nature 05840

62. Dumala N, Mangalampalli B, Kalyan KSS, Grover P. Biochemical alterations induced by nickel oxide nanoparticles in female Wistar albino rats after acute oral exposure. Biomarkers. 2018;23:33-43. doi:10.1080/1354750X.2017.1360943

63. Abdel Aziz I, Zabut B. Determination of blood indices of albino rats treated with aluminum chloride and investigation of antioxidant effects of vitamin E and C. Egypt J Biol. 2011;13:1-7.

64. Sarhan OMM, Hussein RM. Effects of intraperitoneally injected silver nanoparticles on histological structures and blood parameters in the albino rat. Int J Nanomedicine. 2014;9:1505-1517. doi:10.2147/IJN.S56729

65. Li WQ, Wang Z, Hao S, et al. Mitochondria-targeting polydopamine nanoparticles to deliver doxorubicin for overcoming drug resistance. ACS Appl Mater Interfaces. 2017;9:16793-16802. doi:10.1021/ acsami.7b01540

66. Agarwal A, Saleh RA, Bedaiwy MA. Role of reactive oxygen species in the pathophysiology of human reproduction. Fertil Steril. 2003;79:829-843.

67. Kusaczuk M, Kretowski R, Naumowicz M, Stypulkowska A, Cechowska-Pasko M. Silica nanoparticle-induced oxidative stress and mitochondrial damage is followed by activation of intrinsic apoptosis pathway in glioblastoma cells. Int $J$ Nanomedicine. 2018;13:2279-2294. doi:10.2147/IJN.S158393

68. Bustos PL, Volta BJ, Perrone AE, Milduberger N, Bua J. A homolog of cyclophilin D is expressed in Trypanosoma cruzi and is involved in the oxidative stress-damage response. Cell Death Discov. 2017;3:16092. doi:10.1038/cddiscovery.2016.92

69. Lee JE, Park JH, Shin IC, Koh HC. Reactive oxygen species regulated mitochondria-mediated apoptosis in PC12 cells exposed to chlorpyrifos. Toxicol Appl Pharmacol. 2012;263:148-162. doi:10.1016/j.taap.2012.06.005

70. Kanwal U, Bukhari NI, Rana NF, et al. Doxorubicin-loaded quaternary ammonium palmitoyl glycol chitosan polymeric nanoformulation: uptake by cells and organs. Int J Nanomedicine. 2019;14:1-15. doi:10.2147/IJN.S176868 


\section{Publish your work in this journal}

The International Journal of Nanomedicine is an international, peerreviewed journal focusing on the application of nanotechnology in diagnostics, therapeutics, and drug delivery systems throughout the biomedical field. This journal is indexed on PubMed Central, MedLine, CAS, SciSearch ${ }^{\circledR}$, Current Contents ${ }^{\circledR} /$ Clinical Medicine,
Journal Citation Reports/Science Edition, EMBase, Scopus and the Elsevier Bibliographic databases. The manuscript management system is completely online and includes a very quick and fair peer-review system, which is all easy to use. Visit http://www.dovepress.com/ testimonials.php to read real quotes from published authors. 